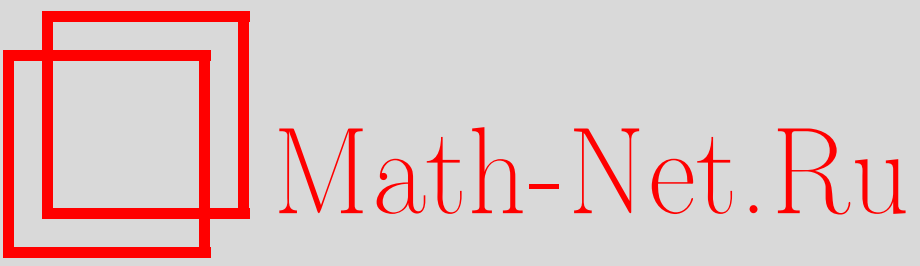

А. В. Нестеров, Об асимптотике решения задачи Коши для сингулярно возмущенной системы уравнений переноса с малой нелинейной диффузией, Итоги науки и техн. Сер. Соврем. мат. и ее прил. Темат. обз., 2021, том 192, 84-93

DOI: https://doi.org/10.36535/0233-6723-2021-192-84-93

Использование Общероссийского математического портала Math-Net.Ru подразумевает, что вы прочитали и согласны с пользовательским соглашением

http://www.mathnet.ru/rus/agreement

Параметры загрузки:

IP: 35.173 .219 .149

26 апреля 2023 г., 03:12:13 


\title{
ОБ АСИМПТОТИКЕ РЕШЕНИЯ ЗАДАЧИ КОШИ ДЛЯ СИНГУЛЯРНО ВОЗМУЩЕННОЙ СИСТЕМЫ УРАВНЕНИЙ ПЕРЕНОСА С МАЛОЙ НЕЛИНЕЙНОЙ ДИФФУЗИЕЙ
}

\author{
(c) 2021 г. $\quad$ A. B. HECTEPOB
}

\begin{abstract}
АннотАция. В работе приведен обзор некоторых асимптотик решений сингулярно возмущенных систем уравнений переноса, а также представлены новые результаты. Особенностью рассмотренных задач является принадлежность к так называемому критическому случаю, когда вырожденное решение является однопараметрическим семейством. При определенных условиях это приводит к быстрому установлению динамического равновесия между компонентами решения и последующему переносу с «осредненной» скоростью. Области больших градиентов начальных условий порождают внутренние слои, которые могут описываться линейными параболическими уравнениями и их обобщениями - уравнениями типа Бюргерса, Бюргерса-Кортевега-де Фриса.
\end{abstract}

Ключевые слова: система уравнений переноса, сингулярное возмущение, асимптотическое разложение по малому параметру, критический случай, параболический переходный слой, уравнение Бюргерса-Кортевега-де Фриса.

\section{ON THE ASYMPTOTICS OF THE SOLUTION \\ TO THE CAUCHY PROBLEM FOR A SINGULARLY PERTURBED SYSTEM OF TRANSFER EQUATIONS WITH LOW NONLINEAR DIFFUSION}

\author{
(C) 2021 A. V. NESTEROV
}

\begin{abstract}
This paper is a survey of results concerning asymptotics of solutions of singularly perturbed systems of transport equations; it also contains some new results. We discuss the so-called critical problems whose degenerate solutions are one-parameter families. Under certain conditions, this leads to a fast establishment of dynamic equilibrium between the components of the solution and the subsequent transfer with an "average" rate. The regions of large gradients of the initial conditions generate inner layers, which can be described by linear parabolic equations and their generalizations, for example, equations of the Burgers and Burgers-Korteweg-de Vries types.
\end{abstract}

Keywords and phrases: system of transport equations, singular perturbation, asymptotic expansion in a small parameter, critical case, parabolic transition layer, Burgers-Korteweg-de Vries equation.

AMS Subject Classification: 35F40, 35F55, 35F61, 35F99

Работа выполнена при поддержке гранта РЭУ им. Г. В. Плеханова по теме «Интеллектуальная система анализа спутниковых данных с целью прогнозирования экономических последствий динамики глобального распределения запасов питьевой воды и пожарной опасности». 
1. Введение. В настоящей работе приведены некоторые результаты исследований сингулярно возмущенных систем уравнений переноса вида

$$
\varepsilon^{2}\left(U_{t}+D U_{x}\right)=A U+\varepsilon^{m} P .
$$

Здесь $\varepsilon$ - малый параметр, $m>0$ - целое, решение $U \in \mathbb{R}^{n}$, матрица $D$ всюду ниже-диагональная, матрицы $D, A$ могут быть постоянными или зависеть от времени и пространственных переменных $(t, x)$, малое слагаемое $P$ в правой части может содержать различного рода нелинейности, диффузионные члены и т. п. В прикладных областях такими системами описываются процессы переноса в многофазных средах в случае, когда процессы обмена между фазами протекают намного быстрее, чем процессы переноса (см. [10]). Особенностью рассматриваемых задач является вырожденность матрицы $A$, а именно, наличие однократного нулевого собственного значения. В этом случае вырожденное решение (при $\varepsilon=\mu=0$ ) определяется неоднозначно. С точки зрения теории сингулярных возмущений это относит задачи к так называемому критическому случаю (см. [2]), с точки зрения приложений такое условие связано с законами сохранения (см. ниже). В упомянутых ниже работах строятся асимптотические разложения по малым параметрам решений начальных и начально-краевых задач для подобного типа систем уравнений. В начале приводится краткий обзор некоторых работ, посвященных исследованиям в данной области.

Всюду ниже мы обозначаем малый положительный параметр греческой буквой $\varepsilon$ и считаем, что $0<\varepsilon \ll 1$. Считаем также, что матрица $A$ удовлетворяет следующему условию.

Условие 1. Матрица $A$ имеет однократное нулевое собственное значение, все остальные собственные значения имеют отрицательные вещественные части. Всюду ниже через $h_{0}, h_{0}^{*}$ обозначены собственные векторы матриц $A, A^{T}$ соответственно (нижний индекс может отсутствовать). Векторы $h_{0}, h_{0}^{*}$ выбраны так, что $\left(h_{0}, h_{0}^{*}\right)=1$.

2. Обзор. В [1] исследовалась начально-краевая задача для сингулярно возмущенной системы уравнений первого порядка

$$
\left\{\begin{array}{l}
\varepsilon\left(u_{t}+\Lambda_{1} u_{x}\right)=-a u+b v, \quad x>0, \quad t>0, \\
\varepsilon\left(v_{t}+\Lambda_{2} v_{x}\right)=\gamma(a u-b v) \\
u(x, 0)=\varphi_{1}(x), \quad v(x, 0)=\varphi_{2}(x) \\
u(0, t)=\psi_{1}(t), \quad v(0, t)=\psi_{2}(t)
\end{array}\right.
$$

где $a, b, \gamma>0$, краевые и начальные значения функций $u$ и $v$, заданные при $x=0$ и $t=0$, согласованы в угловой точке $(0,0): \varphi_{1}(0)=\psi_{1}(0), \varphi_{2}(0)=\psi_{2}(0)$. Решением вырожденной задачи (при $\varepsilon=0$ )

$$
-a u+b v=0
$$

является однопараметрическое семейство

$$
u(x, t, 0)=g(x, t), \quad v(x, t, 0)=\frac{a}{b} g(x, t),
$$

где $g(x, t)$ - произвольная функция. Уравнение для определения функции $g(x, t)$ находится из условий разрешимости системы уравнений, определяющих слагаемые $\bar{u}_{1}, \bar{v}_{1}$ в регулярной части асимптотического разложения решения

$$
\left\{\begin{array}{l}
\bar{u}(x, t, \varepsilon)=\bar{u}_{0}(x, t)+\varepsilon \bar{u}_{1}(x, t)+\ldots, \\
\bar{v}(x, t, \varepsilon)=\bar{v}_{0}(x, t)+\varepsilon \bar{v}_{1}(x, t)+\ldots
\end{array}\right.
$$

Функция $g(x, t)$ есть решение задачи

$$
\left\{\begin{array}{l}
g_{t}+\Lambda_{3} g_{x}=0, \\
g(x, 0)=g 1(x), \quad g(0, t)=g 2(t), \quad g 1(0) \neq g 2(0),
\end{array}\right.
$$

где $g 1(x), g 2(t)$ определенным образом выражаются через начальные и краевые условия исходной задачи,

$$
\Lambda_{3}=\frac{a \Lambda_{2}+\gamma b \Lambda_{1}}{a+\gamma b}
$$


Соответственно, функция $g(x, t)$, а вместе с ней и $\bar{u}_{0}(x, t), \bar{v}_{0}(x, t)($ см. $(2))$ оказываются разрывными на линии $x=\Lambda_{3} t$. Отмечено, что эта линия не совпадает ни с одной характеристикой исходной системы уравнений $(1)$, выходящих из угловой точки $(0,0)$ границы, вместе с тем разрывы точного решения могут распространяться только вдоль характеристик системы (1). В [1] показано, что при $\varepsilon \rightarrow+0$ решение задачи (1) стремится к решению вырожденной задачи всюду, кроме произвольно малых, но фиксированных окрестностей линии $x=\Lambda_{3} t$ и границ области:

$$
\begin{gathered}
\forall \delta>0 \forall t>\delta, x>\delta,\left|x-\Lambda_{3} t\right|>\delta \\
\lim _{\varepsilon \rightarrow+0} u(x, t, \varepsilon)=\bar{u}(x, t), \quad \lim _{\varepsilon \rightarrow+0} v(x, t, \varepsilon)=\bar{v}(x, t) .
\end{gathered}
$$

Из результатов [1] следует, что точное решение в малой окрестности линии $x=\Lambda_{3} t$, оставаясь гладким, имеет характер переходного слоя, который при $\varepsilon \rightarrow+0$ переходит в разрыв. Структура переходного слоя в работе [1] не исследовалась.

В [3] исследовалась начально-краевая задача для системы двух уравнений

$$
\left\{\begin{array}{l}
\varepsilon^{2}\left(u_{t}+u_{x}\right)=-a(x) u+b(x) v, \quad x>0, \quad t>0, \\
\varepsilon^{2} v_{t}=a(x) u-b(x) v, \\
u(x, 0)=\psi(x), \quad u(0, t)=\varphi(t), \quad v(x, 0)=\eta(x) ;
\end{array}\right.
$$

здесь $a(x)>a_{0}>0, b(x)>b_{0}>0$. Начальные и краевые условия могут быть не согласованы в угловой точке $(0,0)$. Показано, что вырожденное решение (при $\varepsilon=0$ ) имеет разрыв на линии

$$
t=B(x)=\int_{0}^{x} a(s) / b(s) d s,
$$

выходящей из угловой точки границы $(0,0)$. Эта линия не совпадает с характеристикой $x=t$ исходной системы (3), выходящей из той же угловой точки $(0,0)$. Построено асимптотическое представление решения, состоящее из сглаженной в окрестности линии разрыва регулярной части, функции переходного слоя и внутреннего разложения в окрестности угловой точки границы $(0,0)$. Решение задачи $(2)$ в малой окрестности линии $t=B(x)$ имеет характер переходного слоя, который описывается в переменных $(t, \varsigma)$ параболическим уравнением с главной частью

$$
\varphi_{t}+M(t) \varphi_{\varsigma \varsigma}, \quad \varsigma=\frac{t-B(x)}{\varepsilon},
$$

где коэффициент $M(t)<0$ выражается через данные задачи. Построено асимптотическое представление решения с точностью $O\left(\varepsilon^{1 / 2} \ln \varepsilon\right)$, доказана равномерная оценка остаточного члена. Показано, что разрыв точного решения на линии $x=t$, обусловленный возможной несогласованностью начальных и краевых данных, асимптотически затухает как $\exp \left(-\varkappa x / \varepsilon^{2}\right), \varkappa>0$, и при любом фиксированном (по отношению к параметру $\varepsilon$ ) $x>0$ становится величиной порядка $O\left(\varepsilon^{N}\right)$ при всех $N>0$. Полученные результаты можно интерпретировать следующим образом. При малых значениях параметра $\varepsilon$ в $\varepsilon^{2}$-окрестностях линии $t=0$ устанавливается динамическое равновесие между компонентами решения, после чего происходит перенос «квазиравновесного» решения с некоторой осредненной скоростью. В области больших градиентов - линии $t=B(x)$, кроме переноса, происходит «псевдодиффузия» с коэффициентом «псевдодиффузии» $M(t)$ в соответствии с уравнением с главной частью (4), хотя исходная система уравнений - гиперболическая.

В [7] исследовалось влияние малой нелинейности, добавленной в правую часть системы уравнений, на переходный слой в решении начально-краевой задачи

$$
\varepsilon^{2}\left(U_{t}+D U_{x}\right)=A U+\varepsilon F(U), \quad x>0, \quad t>0, \quad U(x, 0)=U^{0}(x), \quad U(0, t)=\Phi(t) .
$$

Здесь матрицы $D, A$ постоянные, матрица $A$ удовлетворяет условию 1 . На нелинейность наложены условия: $F(U)$ достаточно гладкая и $\left(F(U), h_{0}^{*}\right)=0$. Начальные и краевые условия могут быть не согласованы в угловой точке $(0,0)$. Показано, что вырожденное решение (при $\varepsilon=0$ ) задачи $(5)$ имеет разрыв на линии $x=V t, V=\left(D h_{0}, h_{0}^{*}\right)$, выходящей из угловой точки границы $(0,0)$. Эта 
линия, вообще говоря, не совпадает ни с одной характеристикой исходной системы (5), выходящих из той же угловой точки $(0,0)$. Построено асимптотическое представление асимптотическое представление решения, состоящее из сглаженной в окрестности линии разрыва регулярной части и функций переходного слоя. Решение задачи (5) в $\varepsilon$-окрестности линии $x=V t$ имеет характер переходного слоя, который описывается нелинейным параболическим уравнением

$$
\varphi_{t}+M \varphi_{\varsigma \varsigma}+\left(F_{\text {eff }}(\varphi)\right)_{\varsigma}^{\prime}=0 .
$$

Здесь $\varsigma=(x-V t) / \varepsilon, M=\left((D-V I) G(D-V I) h_{0}, h_{0}^{*}\right), I$ - единичная матрица, $G$ - псевдообратная к $A$ матрица (если $\left(z, h_{0}^{*}\right)=0$, то решение системы $A x=z$ выражается как $x=G z+C h_{0}$ ), $F_{\text {eff }}(z)$ выражается через данные задачи. Накладывается условие $M<0$, показано, что класс матриц $D, A$, при которых $M<0$ - не пустой. При квадратичной нелинейности $F(U)$ в исходной системе уравнений уравнение (6) становится уравнением Бюргерса. Оценка остаточного члена дана по невязке в области $x \geqslant 0, t \geqslant 0$ всюду, кроме произвольно малой, но фиксированной окрестности точки $(0,0)$.

В [4] исследовалось влияние иной малой нелинейности, добавленной в правую часть сингулярно возмущенной системы

$$
\varepsilon^{2}\left(U_{t}+D U_{x}\right)=A U+\varepsilon^{2} F(U), \quad x>0, \quad t>0 .
$$

Для системы (7) ставились начальные условия вида

$$
U(x, 0)=H \omega\left(\frac{x}{\varepsilon}\right),
$$

где гладкая функция $\omega(z)$ быстро убывает вместе с производными. Построено полное асимптотическое разложение решения, которое имеет вид

$$
U(x, t, \varepsilon)=\sum_{i=0}^{N} \varepsilon^{i}\left(s_{i}(\varsigma, t)+p_{i}(\xi, \tau)\right)+R_{n} .
$$

Здесь

$$
\zeta=\frac{x-V t}{\varepsilon}, \quad \xi=\frac{x}{\varepsilon}, \quad \tau=\frac{t}{\varepsilon^{2}}, \quad V=\left(D h_{0}, h_{0}^{*}\right) .
$$

Порядок разложения $N$ определяется порядком гладкости начальных условий и функции $F$. Для всех членов разложения $s_{i}$ и $p_{i}$ найдены уравнения и начальные условия. Пограничные функции $p_{i}$ строятся стандартным способом (см. [2]) и экспоненциально убывают по растянутой переменной $\tau$

$$
\left|p_{i}(\xi, \tau)\right|<C \exp (-\varkappa \tau), \quad \varkappa>0 .
$$

Главные члены асимптотического разложения имеют вид

$$
U(x, t, \varepsilon)=s_{0}(\varsigma, t)+p_{0}(\xi, \tau)+O(\varepsilon) .
$$

В силу оценки (8) при сколь угодно малых, но фиксированных значениях $t>0$ второе слагаемое в $(9)$ становится асимптотически малым и решение может быть представлено только через нулевую $s$-функцию

$$
U(x, t, \varepsilon)=s_{0}(\varsigma, t)+O(\varepsilon)=h_{0} \varphi(\zeta, t)+O(\varepsilon),
$$

где $\varphi_{0}$ есть решение начальной задачи для квазилинейного параболического уравнения

$$
\varphi_{0, t}+M \varphi_{0, \varsigma \varsigma}+F_{\text {eff }}\left(\varphi_{0}\right)=0
$$

с быстро убывающими начальными условиями

$$
\varphi_{\zeta, 0}=k \omega(\zeta)
$$

здесь $k$-константа, определяемая из условий задачи (7). В уравнении (10) константа $M<0$ и функция $F_{\text {eff }}$ выражаются через данные исходной задачи $(7)$. Доказана теорема о равномерной оценке остаточного члена. 
В [8] исследовано влияние добавления малого слагаемого с вторыми производными (малой диффузии) в правую часть сингулярно возмущенной системы уравнений с начально-краевыми условиями

$$
\left\{\begin{array}{l}
\varepsilon^{2}\left(U_{t}+D(x) U_{x}\right)=A(x) U+\varepsilon^{4} B(x) U_{x x}, \quad x>0, \quad t>0, \\
U(x, 0)=U^{0}(x), \quad U(0, t)=\Phi(t) .
\end{array}\right.
$$

Здесь матрица $B(x)$ - диагональная, $b_{i i} \geqslant 0$ при всех $i=1, \ldots, n$. Общая структура асимптотического представления решения остается такой же, как и в [7], но в уравнении переходного слоя коэффициенты диффузии и «псевдодиффузии» складываются. Главная часть уравнения, описывающего переходный слой, имеет вид

$$
\varphi_{t}+M \varphi_{\varsigma \varsigma}
$$

где $M$ для постоянных $D, A, B$ имеет вид

$$
M=\left((D-V I) G(D-V I) h_{0}, h_{0}^{*}\right)-\left(B h_{0} / V^{2}, h_{0}^{*}\right)
$$

(для переменных матриц $D, A, B$ соответствующая формула выглядит более громоздко; см. [8]).

В [9] исследовалась начальная задача для сингулярно возмущенной системы уравнений переноса с малой нелинейностью в случае двух пространственных переменных.

$$
\left\{\begin{array}{l}
\varepsilon^{2}\left(U_{t}+D_{1} U_{x_{1}}+D_{2} U_{x_{2}}\right)=A U+\varepsilon^{2} F(U), \quad\|\bar{x}\|<\infty, \quad t>0, \\
U(x, 0)=H \omega\left(\frac{x_{1}}{\varepsilon}, \frac{x_{2}}{\varepsilon}\right) .
\end{array}\right.
$$

На матрицы $A, D_{1}, D_{2}$ наложены те же требования, что и выше, начальные условия достаточно гладкие и удовлетворяют оценке

$$
\left|\omega^{(j)}\left(z_{1}, z_{2}\right)\right|<C \exp \left(-\varkappa\left(z_{1}^{2}+z_{2}^{2}\right), \quad \varkappa>0\right.
$$

для достаточно больших $j$. Построено полное асимптотическое разложение решения в виде

$$
U\left(x_{1}, x_{2}, t, \varepsilon\right)=\sum_{i=0}^{N} \varepsilon^{i}\left(s_{i}\left(\zeta_{1}, \zeta_{2}, t\right)+p_{i}\left(\xi_{1}, \xi_{2}, \tau\right)\right)+R_{N} ;
$$

здесь

$$
\zeta_{i}=\frac{x_{i}-V_{i} t}{\varepsilon}, \quad V_{i}=\left(D_{i} h_{0}, h_{0}^{*}\right), \quad \xi_{i}=\frac{x_{i}}{\varepsilon}, \quad i=1,2 ; \quad \tau=\frac{t}{\varepsilon^{2}} .
$$

Получены уравнения и начальные условия для всех членов разложения. В частности,

$$
s_{0}\left(\zeta_{1}, \zeta_{2}, t\right)=h_{0} \varphi_{0}\left(\zeta_{1}, \zeta_{2}, t\right),
$$

где $\varphi_{0}-$ решение задачи Коши для уравнения

$$
\varphi_{0, t}+M_{1} \varphi_{0, \zeta_{1} \zeta_{1}}+M_{2} \varphi_{0, \zeta_{1} \zeta_{2}}+M_{3} \varphi_{0, \zeta_{2} \zeta_{2}}=F_{\text {eff }}\left(\varphi_{0}\right), \quad t>0,
$$

а коэффициенты $M_{i}, i=1,2,3$, и нелинейность $F_{\text {eff }}(z)$ выражаются через данные задачи, на которые накладывается требование, чтобы уравнение (11) было параболическим.

Показано, что квадратичная форма, отвечающая пространственной части уравнения (11), может быть вырожденной, что зависит от $n$ - числа неизвестных (уравнений) в системе. Если $n=2$, то форма полузнакоопределена.

При определенных условиях, наложенных на данные задачи, доказана теорема о равномерной оценке остаточного члена.

В [5] строилось асимптотическое разложение решения начальной задачи для сингулярно возмущенной системы уравнений в случае многих пространственных переменных

$$
\left\{\begin{array}{l}
\varepsilon^{2}\left(U_{t}+\sum_{i=1}^{K} D_{i} U_{x_{i}}\right)=A U+\varepsilon^{2} F(U), \quad t>0, \\
U(\bar{x}, 0)=H \omega\left(\frac{\bar{x}}{\varepsilon}\right) .
\end{array}\right.
$$


Матрицы $D_{i}, A$ удовлетворяют наложенным выше условиям, функция $\omega(z)$ быстро убывает вместе со всеми производными при $\|\bar{z}\| \rightarrow \infty$, функция $F(U)$ достаточно гладкая. Построено полное асимптотическое разложение решения начальной задачи

$$
U\left(x_{1}, x_{2}, t, \varepsilon\right)=\sum_{i=0}^{N} \varepsilon^{i}\left(s_{i}(\bar{\zeta}, t)+p_{i}\left(\bar{\xi}_{i}, \tau\right)\right)+R_{N},
$$

где

$$
\zeta_{i}=\frac{x_{i}-V_{i} t}{\varepsilon}, \quad V_{i}=\left(D_{i} h_{0}, h_{0}^{*}\right), \quad \xi_{i}=\frac{x_{i}}{\varepsilon}, \quad i=1, \ldots, K ; \quad \tau=\frac{t}{\varepsilon^{2}} .
$$

Для всех членов асимптотического разложения получены уравнения и начальные условия. Главный (при произвольных, но фиксированных значениях $t>0$ ) член асимптотического разложения имеет вид $s_{0}(\bar{\zeta}, t)=h_{0} \varphi_{0}(\bar{\zeta}, t)$, где $\varphi_{0}$ - решение уравнения

$$
\varphi_{0, t}+\sum_{i, j=1}^{K} M_{i j} \varphi_{0, \zeta_{i} \zeta_{j}}=F_{\text {eff }}\left(\varphi_{0}\right), \quad t>0,
$$

а коэффициенты $M_{i j}$ и нелинейность $F_{\text {eff }}(z)$ выражаются через данные задачи. Пространственная часть уравнения (12) может иметь разную степень вырождения, что зависит от соотношения между $n$ - числом неизвестных в системе и $K$ - числом пространственных переменных. При определенных условиях доказана теорема о равномерной оценке остаточного члена.

3. Постановка задачи. Настоящая работа является продолжением работ $[7,8]$.

Основной целью ниже изложенного является получение главного члена асимптотического разложения решения задачи Коши для сингулярно возмущенной системы уравнений переноса с малыми нелинейностью и диффузией следующего вида (см. [6]):

$$
\left\{\begin{array}{l}
\varepsilon^{2}\left(U_{t}+D U_{x}\right)=A U+\varepsilon F(U)+\varepsilon^{3} B(U) U_{x x}, \quad|x|<\infty, \quad t>0, \\
U(x, 0)=H \omega\left(\frac{x}{\varepsilon}\right) .
\end{array}\right.
$$

Здесь $U=\left(u_{1}, \ldots, u_{n}\right)$ - решение, $\varepsilon$-малый положительный параметр, $D$ - диагональная матрица, вектор-функция $F(U)$ и матрица $B(U)$ достаточно гладкие, гладкая функция $\omega(z)$ быстро убывает вместе со всеми производными:

$$
\forall k\left|\omega^{(k)}(z)\right|<C \exp \left(-\varkappa z^{2}\right), \quad \varkappa>0 .
$$

Матрица $A$ удовлетворяет условию 1 . Дополнительно требуется, чтобы

$$
\left(F(U), h_{0}^{*}\right)=0, \quad B(U) h_{0}^{*}=0 .
$$

Отметим, что из требования (14) вытекает закон сохранения

$$
\int_{-\infty}^{+\infty}\left(U, h_{0}^{*}\right) d x=\text { const }
$$

который можно назвать законом сохранения «обобщенной массы».

Вырожденное решение задачи (13) (при $\varepsilon=0$ ) определяется из уравнения

$$
A U=0,
$$

и, соответственно, имеет вид $U(x, t, 0)=g(x, t) h_{0}$, где $g(x, t)$ - произвольная функция.

Асимптотическое представление решения задачи (13) строится методом пограничных функций (см. [2]) и имеет вид

$$
U(x, t, \varepsilon)=\sum_{i=0}^{N} \varepsilon^{i}\left(\bar{u}_{i}(x, t)+s_{i}(\zeta, t)+p_{i}\left(\xi_{1}, \tau\right)\right)+R_{N}
$$

здесь

$$
\zeta=\frac{x-V t}{\varepsilon}, \quad \xi=\frac{x}{\varepsilon}, \quad \tau=\frac{t}{\varepsilon^{2}}, \quad V=\left(D h_{0}, h_{0}^{*}\right) .
$$


При начальных условиях выбранного вида слагаемые $\bar{u}_{i}$ играют вспомогательную роль.

В настоящей работе ограничимся получением задач, определяющих $s_{0}, p_{0}$. Поэтому ниже в (15) положим $N=2$, слагаемые с номерами $i=1,2$ играют вспомогательную роль.

Построение членов асимптотического представления подробно описано в $[7,8]$ и др. В соответствии с погранслойным методом А. В. Васильевой и В. Ф. Бутузова (см. [2]) представим нелинейные функции в виде

$$
\begin{aligned}
F(U) & =F(\bar{U}+S+\Pi+R)=F(\bar{U})+(F(\bar{U}+S)-F(\bar{U}))+(F(\bar{U}+\Pi)-F(\bar{U}))+ \\
& +(F(\bar{U}+S+\Pi+R)-F(\bar{U}+S)-F(\bar{U}+\Pi)+F(\bar{U}))=\bar{F}+S F+\Pi F+R F, \\
B(U) & =B(\bar{U}+S+\Pi+R)=B(\bar{U})+(B(\bar{U}+S)-B(\bar{U}))+(B(\bar{U}+\Pi)-B(\bar{U}))+ \\
& +(B(\bar{U}+S+\Pi+R)-F(\bar{U}+S)-B(\bar{U}+\Pi)+B(\bar{U}))=\bar{B}+S B+\Pi B+R B .
\end{aligned}
$$

\section{1. Построение асимптотического представления решения.}

Регулярная часть асимптотического представления строится в виде

$$
\bar{U}(x, t)=\sum_{i=0}^{2} \varepsilon^{i} \bar{u}_{i}(x, t) ;
$$

$\bar{U}$ играет вспомогательную роль и в окончательный вид построенного ниже асимптотического представления решения не входит. Регулярная часть асимптотического представления строится стандартно (см. [2]) и должна удовлетворять регулярной части уравнения и регулярной части начальных условий. Подставив (17) в регулярную часть системы

$$
\varepsilon^{2}\left(\bar{U}_{t}+D \bar{U}_{x}\right)=A \bar{U}+\varepsilon F(\bar{U})+\varepsilon^{3} B(\bar{U}) \bar{U}_{x x},
$$

стандартным способом (см. [2]) получаем уравнения для членов разложения:

$$
\begin{cases}\varepsilon^{0}: & A \bar{u}_{0}=0 \\ \varepsilon^{1}: & A \bar{u}_{1}=-F\left(\bar{u}_{0}\right), \\ \varepsilon^{2}: & A \bar{u}_{2}=\bar{u}_{0, t}-D \bar{u}_{0, x x}-\bar{F}_{u}\left(\bar{u}_{0}\right) \bar{u}_{1} .\end{cases}
$$

Уравнение при $\varepsilon^{1}$ разрешимо в силу условия $\left(F(U), h_{0}^{*}\right)=0$. Отсюда

$$
\bar{u}_{0}=h_{0} u_{0}(x, t), \quad \bar{u}_{1}=h_{0} u_{1}(x, t)-G F\left(\bar{u}_{0}\right) .
$$

Здесь $G$-псевдообратный к $A$ оператор (если в системе $A x=f$ правая часть удовлетворяет условию $\left(f, h^{*}\right)=0$, то $x=G f+c h$, где $h, h^{*}$ - собственные векторы матриц $A$ и $A^{T}$, отвечающие $\lambda=0), u_{0}$ и $u_{1}$ - некоторые скалярные функции. Записав условие разрешимости уравнения при $\varepsilon^{2}$, с учетом условия () получаем уравнение для определения $u_{0}$ :

$$
u_{0, t}+V u_{0, x}=0, \quad V=\left(D h_{0}, h_{0}^{*}\right) .
$$

При поставленных начальных условиях, зависящих только от растянутой переменной $x / \varepsilon$, начальные условия для регулярной части - нулевые $u_{0}(x, 0)=0$, соответственно, $u_{0}(x, t)=0$.

Построение $S$-функиий. Для удовлетворения начальным условиям, зависящим от растянутой переменной $x / \varepsilon$, строится $S$-функция, зависящая от переменной $t$ и растянутой переменной $\zeta$

$$
S(\zeta, t)=\sum_{i=0}^{2} \varepsilon^{i} s_{i}(\zeta, t), \quad \zeta=\frac{x-V t}{\varepsilon},
$$

здесь функции $s_{1}, s_{2}$ играют вспомогательную роль. Функция $S$ есть решение системы

$$
\varepsilon^{2}\left(S_{t}+D S_{x}\right)=A S+\varepsilon S F+\varepsilon^{3} S B(S) S_{x x}, \quad|x|<\infty, \quad t>0 .
$$

Переходя к переменным $(\zeta, t)$, с учетом $\bar{U}=0$ получаем

$$
\varepsilon^{2} S_{t}+\varepsilon(D-V) S_{\zeta}=A S+\varepsilon F(S)+\varepsilon B(S) S_{\zeta \zeta}, \quad|\zeta|<\infty, \quad t>0 .
$$


Подставив (18) в (19), стандартным способом (см. [2]) получаем уравнения для членов разложения:

Отсюда

$$
\begin{cases}\varepsilon^{0}: & A s_{0}=0 \\ \varepsilon^{1}: & A s_{1}=(D-V) s_{0, \zeta}-F\left(s_{0}\right)-B\left(s_{0}\right) s_{0, \zeta \zeta}, \\ \varepsilon^{2}: & A s_{2}=(D-V) s_{1, \zeta}-F^{\prime}\left(s_{0}\right) s_{1}-\left(B^{\prime}\left(s_{0}\right) s_{1} s_{0, \zeta \zeta}+B\left(s_{0}\right) s_{1, \zeta \zeta}\right) .\end{cases}
$$

$$
\left\{\begin{array}{l}
s_{0}=h_{0} \varphi_{0}(x, t), \\
s_{1}=h_{0} \varphi_{1}(x, t)+G\left((D-V) h_{0} \varphi_{0, \zeta}-F\left(h_{0} \varphi_{0}\right)-B\left(\bar{h}_{0} \varphi_{0}\right) h_{0} \varphi_{0, \zeta \zeta}\right) .
\end{array}\right.
$$

Легко показать, что в силу условий (14) система уравнений для определения $s_{1}$ разрешима.

Записав условие разрешимости системы уравнений для $s_{2}$, исключив из него $s_{1}$ и, учтя требования (14), получим уравнение для определения $\varphi_{0}$ :

где

$$
\varphi_{0, t}+M \varphi_{0, \zeta \zeta}+\left(F_{\mathrm{eff}}\left(\varphi_{0}\right)\right)_{\zeta}^{\prime}+\left(B_{\mathrm{eff}}\left(\varphi_{0}\right) \varphi_{0, \zeta \zeta}\right)_{\zeta}^{\prime}=0
$$

$$
\begin{aligned}
M & =\left((D-V) G(D-V) h_{0}, h_{0}^{*}\right), \\
F_{\text {eff }}\left(\varphi_{0}\right) & =-\left((D-V) G F\left(h_{0} \varphi_{0}\right), h_{0}^{*}\right), \\
B_{\text {eff }}\left(\varphi_{0}\right) & =-\left((D-V) G B\left(h_{0} \varphi_{0}\right) h_{0}, h_{0}^{*}\right) .
\end{aligned}
$$

Наложим условие $M<0$. В развернутой форме уравнение (21) имеет вид

$$
\varphi_{0, t}+M \varphi_{0, \zeta \zeta}+F_{\text {eff }}^{\prime}\left(\varphi_{0}\right) \varphi_{0, \zeta}^{\prime}+B_{\text {eff }}\left(\varphi_{0}\right) \varphi_{0, \zeta \zeta \zeta}+B_{\text {eff }}^{\prime}\left(\varphi_{0}\right) \varphi_{0, \zeta \zeta}=0 .
$$

Это уравнение можно назвать обобщенным уравнением Бюргерса-Кортевега-де Фриса (см. [11]); действительно, при квадратичной функции $F(U)$ и постоянной матрице $B(U)$ уравнение (22) приобретает вид

$$
\varphi_{0, t}+M \varphi_{0, \zeta \zeta}+k \varphi_{0} \varphi_{0, \zeta}^{\prime}+B \varphi_{0, \zeta \zeta \zeta}=0
$$

который отличается от уравнения Бюргерса-Кортевега-де Фриса только численными значениями коэффициентов. Начальные условия для функции $\varphi_{0}$ ставятся ниже.

Построение П-функций. Функция

$$
s_{0}(\zeta, t)=h_{0} \varphi_{0}(\zeta, t)
$$

при $t=0$ равна $h_{0} \varphi_{0}(x / \varepsilon, 0)$ и не может удовлетворить начальным условиям

$$
U(x, 0)=H \omega\left(\frac{x}{\varepsilon}\right) .
$$

Для удовлетворения начальных условий строится пограничная функция $\Pi(\xi, \tau)$, зависящая от растянутых переменных $\xi=x / \varepsilon, \tau=t / \varepsilon^{2}$, которая является решением системы

$$
\varepsilon^{2}\left(\Pi_{t}+D \Pi_{x}\right)=A \Pi+\varepsilon \Pi F(U)+\varepsilon^{4} \Pi B \Pi_{x x}, \quad|x|<\infty, \quad t>0 .
$$

Кроме того, пограничная функция П $(\xi, \tau)$ должна совместно с функцией $S$ удовлетворять начальным условиям, а также условиям на бесконечности

$$
S(\zeta, 0)+\Pi(\xi, 0)=H \omega(x / \varepsilon), \quad \Pi(\xi, \tau) \rightarrow 0, \quad \tau \rightarrow+\infty .
$$

Пограничная функция П $(\xi, \tau)$ строится стандартным образом (см. [2]) в виде

$$
\Pi(\xi, \tau, \varepsilon)=p_{0}(\xi, \tau)+R \Pi .
$$

Построение уравнений для $p_{0}$ также стандартно и здесь не описывается; $p_{0}$ определяется как решение системы обыкновенных дифференциальных уравнений

$$
p_{0, \tau}=A p_{0} .
$$

Из (23) получаются начальные условия для функций $s_{0}$ и $p_{0}$, которые определяются совместно, с добавлением условия стремления $p_{0}$ к нулю при $\tau \rightarrow \infty$ :

$$
h_{0} \varphi_{0}(\zeta, 0)+p_{0}(\xi, 0)=H \omega(x / \varepsilon), \quad p_{0}(\xi, \tau) \rightarrow 0, \quad \tau \rightarrow \infty .
$$


Из (26) однозначно определяются начальные условия для функций $p_{0}(\xi, 0)=P \omega(\xi), P$ - вектор, и для $\varphi_{0}(\zeta, 0)=k \omega(\zeta), k$-константа. Решение задачи, определяющей $p_{0}$, а именно системы $(25)$ с условиями (26) существует и удовлетворяет оценке

$$
\left|p_{0}(\xi, \tau)\right|<C \exp (-\varkappa \tau), \quad \varkappa>0 .
$$

Окончательный вид построенного асимптотического представления решения таков:

$$
\left.U(x, t, \varepsilon)=h_{0} \varphi(\zeta, t)+p_{0}\left(\xi_{1}, \tau\right)\right)+R_{0},
$$

где переменные $\zeta, \xi, \tau$, константа $V$ и задачи для определения функций $\varphi_{0}, p_{0}$ определены выше.

3.2. Оценка остаточного члена. Оценка остаточного члена проводилась по невязке. В силу алгоритма построения асимптотического представления и оценки (27) функции $p_{0}$ решение задачи (13) представимо в виде $(28)$, где $\varphi_{0}(\zeta, t), p_{0}(\xi, \tau)$ - построенные выше функции, а остаточный член $R_{0}$ удовлетворяет задаче Коши

$$
\left\{\begin{array}{l}
\varepsilon^{2}\left(R_{0, t}+D R_{0, x}\right)=A R_{0}+\varepsilon R F+\varepsilon^{3} R B R_{0, x x}+r(x, t), \quad|x|<\infty, \quad t>0, \\
R_{0}(x, 0)=0,
\end{array}\right.
$$

где $R F, R B$ определяются формулами (16). Существует такое $T>0$, не зависящее от $\varepsilon$, что $|r|=O(\varepsilon)$ при всех $0<t<T$.

3.3. Выводы. 1. Решение задачи (13) в силу оценки (27) при сколь угодно малых, но фиксированных значениях $t>0$ представимо в виде

$$
U(x, t, \varepsilon)=s_{0}(\zeta, t)+O(\varepsilon)=h_{0} \varphi_{0}(\zeta, t),
$$

где $\varphi_{0}(\zeta, t)$ - решение уравнения

$$
\varphi_{0, t}+M \varphi_{0, \zeta \zeta}+\left(F_{\mathrm{eff}}\left(\varphi_{0}\right)\right)_{\zeta}^{\prime}+\left(B_{\mathrm{eff}}\left(\varphi_{0}\right) \varphi_{0, \zeta \zeta}\right)_{\zeta}^{\prime}=0
$$

В развернутой форме уравнение (30) имеет вид

$$
\varphi_{0, t}+M \varphi_{0, \zeta \zeta}+F_{\text {eff }}^{\prime}\left(\varphi_{0}\right) \varphi_{0, \zeta}^{\prime}+B_{\text {eff }}\left(\varphi_{0}\right) \varphi_{0, \zeta \zeta \zeta}+B_{\text {eff }}^{\prime}\left(\varphi_{0}\right) \varphi_{0, \zeta \zeta}=0
$$

(оно является обобщенным уравнением Бюргерса-Кортевега-де Фриса; см. [11]). При квадратичной функции $F(u)$ и постоянной матрице $B(u)$ уравнение приобретает вид

$$
\varphi_{0, t}+M \varphi_{0, \zeta \zeta}+k \varphi_{0} \varphi_{0, \zeta}^{\prime}+B \varphi_{0, \zeta \zeta \zeta}=0,
$$

которое отличается от уравнения Бюргерса-Кортевега-де Фриса только числовыми значениями коэффициентов.

Известно (см. [11]), что уравнение Бюргерса-Кортевега-де Фриса может иметь решение в виде бегущей волны

$$
\varphi(\zeta, t)=v(\zeta-c t)=v\left(\frac{x-V t}{\varepsilon}-c t\right)=v\left(\frac{x-(V+c \varepsilon) t}{\varepsilon}\right) .
$$

Это, в свою очередь, говорит о том, что в многофазной среде «эффективная» скорость распространения уединенных волн может быть равной $V+\varepsilon c$.

2. При достаточной гладкости входных данных задачи (13) можно построить остальные члены асимптотического разложения (15).

3. Аналогично можно построить асимптотическое разложение решения задачи (13) в случае двух и большего числа пространственных переменных. 
4. Итоги. Особенностью всех рассмотренных выше задач является сингулярное возмущение главной части системы уравнений переноса в сочетании с вырожденностью матрицы $A$. Наличие однократного нулевого собственного значения у матрицы $A$ (при отрицательных вещественных частях остальных) в сочетании с малым параметром при главной части влечет за собой «псевдодиффузионный» характер решения гиперболической системы уравнений.

Можно качественно описать поведение решения во всех рассмотренных выше задачах. В случае начальных задач в окрестности $t=0$ происходят быстрые процессы установления «квазиравновесия» между компонентами решения, что описывается погранфункциями П. Регулярная («квазиравновесная») часть решения $\bar{U}$ распространяется с эффективной - усредненной скоростью переноса вдоль «псевдохарактеристик». Области больших градиентов - внутренние слои, которые описываются функциями $S$, переносятся вдоль тех же «псевдохарактеристик», одновременно с «псевдодиффузионным» размытием. Коэффициент «псевдодиффузии» областей с большими градиентами выражается через скорости переноса компонентов и матрицу $A$. Структура уравнения, определяющего внутренние слои, существенно зависит от малых добавок в правой части системы. Для начально-краевых задач поведение решения качественно имеет такой же характер.

При наличии нулевых корней у матрицы $A$ большей кратности структура асимптотики (и, соответственно, решения) становится существенно иной.

\section{СПИСОК ЛИТЕРАТУРЫ}

1. Васильева А. Б. О внутреннем переходном слое в решении системы уравнений в частных производных первого порядка// Диффер. уравн. - 1985. - 21, № 9. - С. 1537-1544.

2. Васильева А. Б., Бутузов В. Ф. Сингулярно возмущенные уравнения в критических случаях. - М.: Изд-во МГУ, 1978.

3. Нестеров А. В. Об асимптотике с переходным слоем решения одной сигнгулярно возмущенной гиперболической системы уравнений// Докл. АН СССР. - 1989. - 305, № 6. - С. 1350-1353.

4. Нестеров А. В. Об асимптотике решения сингулярно возмущенной гиперболической системы уравнений с малой нелинейностью в критическом случае// Ж. вычисл. мат. мат. физ. -2012 . -52 , № 7 . C. $1035-1043$.

5. Нестеров А. В. О структуре решения одного класса гиперболических систем с несколькими пространственными переменными в дальней зоне// Ж. вычисл. мат. мат. физ. - 2016. - 56, № 4. - С. 639-649.

6. Нестеров А. В. Асимптотика решения задачи Коши для сингулярно возмущенной системы уравнений переноса с малой диффузией// в кн.: Современные методы теории краевых задач/ Мат. Междунар. конф. «Воронежская весенняя математическая школа «Понтрягинские чтения-XXX» (3-9 мая 2019 г., Воронеж). - Воронеж, 2019. - C. 212-213.

7. Нестеров А. В., Шулико О. В. Асимптотика решения сингулярно возмущенной системы дифференциальных уравнений первого порядка в частных производных с малой нелинейностью в критическом случае// Ж. вычисл. мат. мат. физ. - 2007. - 47, № 3. - С. 438-444.

8. Нестеров А. В., Шулико О. В. Об асимптотике решения сингулярно возмущенной системы параболических уравнений в критическом случае// Ж. вычисл. мат. мат. физ. -2010 . - 50, № 2. - С. 268-275.

9. Нестеров А. В., Павлюк Т. В. Об асимптотике решения сингулярно возмущенной гиперболической системы с несколькими пространственными переменными в критическом случае// Ж. вычисл. мат. мат. физ. - 2014. - 54, № 3. - С. 450-462.

10. Рачинский В. В. Введение в общую теорию динамики сорбции и хроматографии. - М.: Наука, 1964.

11. Уизем Дж. Линейные и нелинейные волны. - М.: Мир, 1977.

Нестеров Андрей Владимирович

Российский экономический университет им. Г. В. Плеханова, Москва

E-mail: andrenesterov@yandex.ru 